\title{
Revisiting Hammerstein system identification through the Two-Stage Algorithm for bilinear parameter estimation
}

Jiandong Wang, Qinghua Zhang, Lennart Ljung

Division of Automatic Control

E-mail: jiandong@pku.edu.cn, zhang@irisa.fr,

ljung@isy.liu.se

20th December 2010

Report no.: LiTH-ISY-R-2984

Accepted for publication in Automatica, Vol 45, pp 2627-2633, 2009.

Address:

Department of Electrical Engineering

Linköpings universitet

SE-581 83 Linköping, Sweden

WWW: http://WwW.control.isy.liu.se

AUTOMATIC CONTROL

REGLERTEKNIK

LINKÖPINGS UNIVERSITET

Technical reports from the Automatic Control group in Linköping are available from http://www. control.isy.liu.se/publications. 


\begin{abstract}
The Two-Stage Algorithm (TSA) has been extensively used and adapted for the identification of Hammerstein systems. It is essentially based on a particular formulation of Hammerstein systems in the form of bilinearly parameterized linear regressions. This paper has been motivated by a somewhat contradictory fact: though the optimality of the TSA has been established by Bai in 1998 only in the case of some special weighting matrices, the unweighted TSA is usually used in practice. It is shown in this paper that the unweighted TSA indeed gives the optimal solution of the weighted nonlinear least squares problem formulated with a particular weighting matrix. This provides a theoretical justification of the unweighted TSA, and also leads to a generalization of the obtained result to the case of colored noise with noise whitening. Numerical examples of identification of Hammerstein systems are presented to validate the theoretical analysis.
\end{abstract}

Keywords: System Identification 
Brief paper

\title{
Revisiting Hammerstein system identification through the Two-Stage Algorithm for bilinear parameter estimation ${ }^{\text {th }}$
}

\author{
Jiandong Wang ${ }^{\mathrm{a}}$, Qinghua Zhang ${ }^{\mathrm{b}, *}$, Lennart Ljung ${ }^{\mathrm{c}}$ \\ ${ }^{a}$ College of Engineering, Peking University, Beijing 100871, China \\ ${ }^{\mathrm{b}}$ INRIA, Campus de Beaulieu, 35042 Rennes Cedex, France

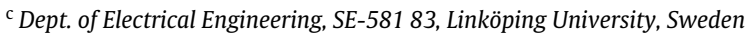

\section{A R T I C L E I N F O}

\section{Article history:}

Received 8 December 2008

Received in revised form

29 April 2009

Accepted 14 July 2009

Available online 17 September 2009

\section{Keywords:}

Nonlinear system identification

Hammerstein system

Two-stage algorithm

Bilinear equation

\begin{abstract}
A B S T R A C T
The Two-Stage Algorithm (TSA) has been extensively used and adapted for the identification of Hammerstein systems. It is essentially based on a particular formulation of Hammerstein systems in the form of bilinearly parameterized linear regressions. This paper has been motivated by a somewhat contradictory fact: though the optimality of the TSA has been established by Bai in 1998 only in the case of some special weighting matrices, the unweighted TSA is usually used in practice. It is shown in this paper that the unweighted TSA indeed gives the optimal solution of the weighted nonlinear least squares problem formulated with a particular weighting matrix. This provides a theoretical justification of the unweighted TSA, and also leads to a generalization of the obtained result to the case of colored noise with noise whitening. Numerical examples of identification of Hammerstein systems are presented to validate the theoretical analysis.
\end{abstract}

(c) 2009 Elsevier Ltd. All rights reserved.

\section{Introduction}

Within the class of block-oriented nonlinear systems, a Hammerstein system is composed of a static nonlinearity block followed by a linear dynamic block. Typically, the nonlinearity of such a system is caused by actuator distortions. Many practical processes can also be effectively modeled as Hammerstein systems, for example, heat exchangers (Eskinat, Johnson, \& Luyben, 1991), electrical drives (Balestrino, Landi, Ould-Zmirli, \& Sani, 2001), thermal microsystems (Sung, 2002), physiological systems (Dempsey \& Westwick, 2004), sticky control valves (Srinivasan, Rengaswamy, Narasimhan, \& Miller, 2005), solid oxide fuel cells (Jurado, 2006), and magneto-rheological dampers (Wang, Sano, Chen, \& Huang, 2009).

In the quite vast literature on Hammerstein systems, most known identification methods are covered by the ten methods summarized in Section 3.9 of Haber and Keviczky (1999). Another

\footnotetext{
The work was partially supported by the National Natural Science Foundation of China under grants No.60704031 and No.10832006. The material in this paper was partially presented at SYSID 2009, Saint Malo, July 2009. This paper was recommended for publication in revised form by Associate Editor Er-Wei Bai under the direction of Editor Torsten Söderström.

* Corresponding author. Tel.: +33 29984 7463; fax: +33 299847171.

E-mail address: zhang@irisa.fr (Q. Zhang).
}

quite complete survey given in Chapter 1 of Janczak (2005) classifies most existing methods into four groups. The present paper focuses on the so-called Two-Stage Algorithm (TSA), also known as the over-parameterized method. It has been extensively used and adapted in the identification of block-oriented nonlinear systems, e.g., for Hammerstein-Wiener systems (Abrahamsson, Kay, \& Stoica, 2007; Bai, 1998; Mzyk, 2000; Palanthandalam-Madapusi, Bernstein, \& Ridley, 2006; Palanthandalam-Madapusi, Ridley, \& Bernstein, 2005), and for Hammerstein or Wiener systems (Chang \& Luus, 1971; Gomez \& Baeyens, 2004, 2005; McKelvey \& Hanner, 2003).

The TSA is essentially based on a particular formulation of the Hammerstein system as a linear regression parameterized in a bilinear form, also referred to as bilinear equation (see, e.g., Cohen and Tomasi (1997) and Bai and Liu (2006)). More specifically, let $\tilde{\Phi}(t) \in \mathbb{R}^{n \times m}$ be a matrix filled with $l=n m$ regressors, the linear regression

$y(t)=b^{T} \tilde{\Phi}(t) a+v(t)$

bilinearly parameterized by $a \in \mathbb{R}^{m}$ and $b \in \mathbb{R}^{n}$ can be used to formulate a Hammerstein system with $y(t) \in \mathbb{R}$ and $v(t) \in \mathbb{R}$ corresponding respectively to the output and to the additive noise of the system (more details will be given in the next section). The estimation of the bilinear parameters $a$ and $b$ is usually formulated as a nonlinear Least Squares (LS) problem. The TSA uses a relaxation approach to solve this problem, by first over-parameterizing the 
bilinearly parameterized model (1) with linear parameters before reducing the estimated linear parameters back to the bilinear parameters $a$ and $b$.

The parameter estimation problem for bilinear equations in the form of (1) is receiving an increasing attention. Cohen and Tomasi (1997) made some preliminary remarks on the problem of solving systems of bilinear equations. The TSA was firstly formulated by Chang and Luus (1971) and was later studied by Bai (1998) regarding its optimality in the sense of a weighted nonlinear LS criterion (to be revisited in Section 3). Goethals, Pelckmans, Suykens, and Moor (2005) applied the equivalence of the TSA in Bai (1998) to an LS support vector machine context for identification of Hammerstein systems. Bai and Liu (2006) compared the normalized iterative method, the TSA, and the application of some numerical search method for nonlinear LS solution. Abrahamsson et al. (2007) proposed a new method to estimate the parameters of a general bilinear equation based on a better approximation of a weighting matrix occurring in the LS problem, with applications to submarine detection and Hammerstein-Wiener model identification. Despite these works, the study of the problem is far from mature, just like stated in Abrahamsson et al. (2007): "Bilinear systems of equations and models, however, are still not very well understood even though they are fairly common and despite the fact that they could be considered the next logical step after linear models." As a matter of fact, the TSA has not been very well understood, for some important questions have not been answered and need further investigations.

In this paper, we will study a somewhat contradictory fact: though the optimality of the TSA has been established in Bai (1998) only in the case of some special weighting matrices, this algorithm, adopted in some other works (e.g., Gomez and Baeyens (2004, 2005), McKelvey and Hanner (2003), Mzyk (2000), Palanthandalam-Madapusi et al. (2006, 2005)), usually uses the unweighted LS solution in its first stage; in other words, the identity matrix, which usually does not belong to the aforementioned special weighting matrices, is used. The contribution of this paper is to resolve this "contradiction" by justifying the use of unweighted LS solution in this case, and to generalize the obtained result to the case of colored noise with noise whitening. That is, this paper enhances and extends the result in Bai (1998) by showing that for any arbitrary weighting matrix used in the TSA, this algorithm is also optimal, but for a nonlinear LS criterion formulated with another weighting matrix.

The rest of the paper is organized as follows. Section 2 describes the bilinear equation for Hammerstein systems. The main contribution of the paper is presented in Section 3 in the process of answering optimality questions of the TSA; selection of the weighting matrix for colored noises is discussed therein as well. Numerical examples of identification of Hammerstein systems are presented in Section 4 to validate the theoretical analysis. Section 5 concludes the paper.

\section{Bilinear equation formulation of Hammerstein systems}

It will be shown in this section that, when appropriately parameterized, Hammerstein system identification can be formulated in the form of (1), which is a linear regression with bilinear parameters. In order to focus on the main issues of this paper, let us consider a single-input and single-output discrete-time Hammerstein system with a finite impulse response (FIR) linear part, as illustrated in Fig. 1 where $u(t)$ and $y(t)$ are respectively the input and the noise-corrupted output of the Hammerstein system. The case of infinite impulse response linear part can be addressed similarly using the separable LS technique (see, e.g., Ljung (1999)); the details are omitted due to space limitation.

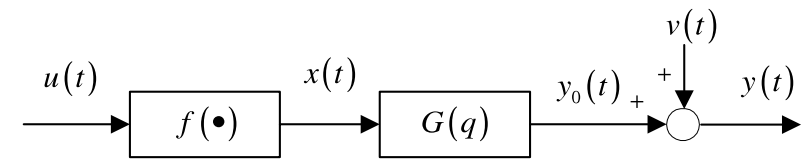

Fig. 1. A discrete-time Hammerstein model.

More precisely, the FIR linear part $G(q)$ in Fig. 1 is parameterized by $b_{1}, \ldots, b_{n}$ such that

$y(t)=\sum_{i=1}^{n} b_{i} x(t-i)+v(t)$

The additive noise $v(t)$ of zero mean is assumed to be white for the time being, whereas the case of colored noises is discussed later in Section 3.3 and Example 2. The static nonlinearity $f(\cdot)$ is assumed to be a linear combination of known basis functions $\phi_{i}(\cdot)$,

$x(t)=\sum_{j=1}^{m} a_{j} \phi_{j}(u(t))$.

Here the orders $n$ and $m$ are assumed to be known $a$ prior Substituting the unmeasurable inner signal $x(t)$ in (3) into (2) yields a linear regression model parameterized in a bilinear form,

$$
\begin{aligned}
y(t)= & {\left[\begin{array}{c}
b_{1} \\
b_{2} \\
\vdots \\
b_{n}
\end{array}\right]^{T}\left[\begin{array}{ccc}
\phi_{1}(u(t-1)) & \cdots & \phi_{m}(u(t-1)) \\
\phi_{1}(u(t-2)) & \cdots & \phi_{m}(u(t-2)) \\
\vdots & \ddots & \vdots \\
\phi_{1}(u(t-n)) & \cdots & \phi_{m}(u(t-n))
\end{array}\right]\left[\begin{array}{c}
a_{1} \\
a_{2} \\
\vdots \\
a_{m}
\end{array}\right] } \\
& +v(t) \\
= & : b^{T} \tilde{\Phi}(t) a+v(t) .
\end{aligned}
$$

Let us introduce a notation useful for a different formulation of the bilinearly parameterized linear regression. For any matrix $M=\left[\begin{array}{llll}M_{1} & M_{2} & \cdots & M_{m}\end{array}\right]$, the overlined notation $\bar{M}$ denotes the column vector obtained by stacking the columns $M_{1}, M_{2}, \ldots, M_{m}$. With this notation, (4) can also be written as

$y(t)=\overline{\tilde{\Phi}(t)}^{T} \overline{b a^{T}}+v(t)$.

For an input-output data set $\{u(t), y(t)\}_{t=1}^{N}$, define

$Y=\left[\begin{array}{c}y(1) \\ y(2) \\ \vdots \\ y(N)\end{array}\right], \quad \Phi=\left[\begin{array}{c}\overline{\tilde{\Phi}(1)}^{T} \\ \tilde{\Phi}(2)^{T} \\ \vdots \\ \overline{\tilde{\Phi}(N)}^{T}\end{array}\right], \quad V=\left[\begin{array}{c}v(1) \\ v(2) \\ \vdots \\ v(N)\end{array}\right]$,

then (5) leads to

$Y=\Phi \overline{b a^{T}}+V$.

It is clear that these new notations are such that $Y \in \mathbb{R}^{N}, \Phi \in \mathbb{R}^{N \times l}$ and $V \in \mathbb{R}^{N}$ with $l=m n$.

Now let us introduce a standard assumption to remove the scale ambiguity between $a$ and $b$.

Assumption 1. The first non-zero entry of $b$ is positive, and

$\|b\|_{2}^{2}=b^{T} b=1$.

With the above formulation, the identification of the Hammerstein system amounts to the estimation of the bilinear parameters $a$ and $b$ from the data set $\{u(t), y(t)\}_{t=1}^{N}$.

\section{The TSA and optimality results}

In this section we analyze some optimality properties of the Two-Stage Algorithm (TSA) after a recall of the algorithm. 


\subsection{Weighted least squares criteria and the TSA}

For the estimation of the bilinear parameters $a$ and $b$ in (7), a traditional approach is to consider the weighted nonlinear LS criterion

$$
\min _{b, a} L(b, a, W)=\min _{b, a} \frac{1}{2}\left(\Phi \overline{b a^{T}}-Y\right)^{T} W\left(\Phi \overline{b a^{T}}-Y\right),
$$

where $W \in \mathbb{R}^{N \times N}$ is some chosen symmetric weighting matrix. To ensure the uniqueness of its solution, this LS problem should be solved under the constraints stated in Assumption 1. Since the TSA described later in this section provides a solution naturally satisfying these constraints, we will not always explicitly mention these constraints when the nonlinear LS problem (9) is referred to. The following assumption is also for ensuring the uniqueness of this nonlinear LS solution.

Assumption 2. The matrices $\Phi \in \mathbb{R}^{N \times l}$ and $W \in \mathbb{R}^{N \times N}$ are such that $\Phi^{T} W \Phi$ has full rank.

Remark that this assumption implies that $\Phi$ has full column rank, a condition related to the excitation property of the system input. A positive definite weighting matrix $W$ then ensures Assumption 2 . Some positive semidefinite matrices $W$ can also satisfy this requirement.

Let us slightly reformulate the nonlinear LS problem (9) in order to make the connection with the TSA. By introducing the notation

$\Theta:=b a^{T}$,

the LS problem (9) is equivalent to the following one with a rank constraint,

$$
\begin{gathered}
\min _{\Theta} L(\Theta, W)=\min _{\Theta} \frac{1}{2}(\Phi \bar{\Theta}-Y)^{T} W(\Phi \bar{\Theta}-Y) \\
\text { s.t. } \operatorname{rank}(\Theta)=1 .
\end{gathered}
$$

Some constraints equivalent to those of Assumption 1 should also be added for a unique solution of this LS problem.

Remark that now in (10) the single parameter vector $\theta:=\bar{\Theta}$ shows up quadratically in the weighted LS problem; however, the associated matrix $\Theta \in \mathbb{R}^{n \times m}$ has a rank constraint ${ }^{1}$ which is usually difficult to be taken into account in optimization problems. In this paper we are interested in a solution known as the TSA based on a relaxation approach. It first solves the unconstrained LS problem (10a) by omitting the rank constraint (10b) and then subsequently projects the solution onto the class of bilinear equations via the singular-value decomposition (SVD). The following description of the TSA closely follows that in Bai (1998).

\section{The Two-Stage Algorithm (TSA)}

1. Choose a weighting matrix $W \in \mathbb{R}^{N \times N}$ and use it to estimate the parameter vector $\theta=\bar{\Theta}$ through the solution of the unconstrained weighted linear LS problem (10a), namely,

$$
\hat{\theta}(W)=\left(\Phi^{T} W \Phi\right)^{-1} \Phi^{T} W Y .
$$

2. Build the matrix $\hat{\Theta}(W) \in \mathbb{R}^{n \times m}$ from the vector $\hat{\theta}(W) \in \mathbb{R}^{l}$ such that

$$
\overline{\hat{\Theta}(W)}=\hat{\theta}(W) \text {. }
$$

\footnotetext{
1 The rank constraint was also referred to as the collinearity constraint in Goethals et al. (2005).
}

Let $\sigma_{1}$ be the largest singular value of the matrix $\hat{\Theta}(W)$, and $u_{1}$ and $v_{1}$ be its left and right singular vectors associated with $\sigma_{1}$, then the bilinear parameters $a$ and $b$ are estimated by

$$
\begin{aligned}
& \hat{\hat{a}}(W)=s_{1} v_{1}, \\
& \hat{\hat{b}}(W)=s_{1} \sigma_{1} u_{1},
\end{aligned}
$$

where $s_{1}= \pm 1$ is the sign of the first non-zero entry of $u_{1}$.

\section{Notes on Notations.}

- The solution of the nonlinear LS problem (9) under Assumptions 1 and 2 will be noted as $\hat{a}(W)$ and $\hat{b}(W)$.

- The solution $\Theta$ of the unconstrained weighted linear LS problem (10a), vectorized as $\theta=\bar{\Theta}$, will be noted as $\hat{\theta}(W)$.

- The result of the TSA will be noted as $\hat{\hat{a}}(W)$ and $\hat{\hat{b}}(W)$.

In these notations the weighting matrix $W$ is explicitly indicated, as its different choices will take an important role in this paper.

The TSA is based on a relaxation approach. It first solves the unconstrained LS problem (10a) by omitting the rank constraint (10b) and then subsequently projects the solution onto the class of bilinear equations via the singular-value decomposition (SVD). Though in principle the TSA can provide estimates of $a$ and $b$ for any weighting matrix $W$, it is not obvious if these estimates are the solution of the original LS problem (9) or if they minimize another error criterion. Hence, the significance of the solution given by the TSA is the main question this paper attempts to answer.

This question has already been partly answered by Theorem 2.2 of Bai (1998) which is restated as follows.

Theorem 1. If the weighting matrix $W$, used both in the nonlinear $L S$ problem (9) and in the TSA, satisfies

$\Phi^{T} W \Phi=\alpha I_{l}$,

where $\alpha$ is any positive scalar and $I_{l}$ is the $l \times$ lidentity matrix, then the TSA produces the optimal solution of the nonlinear LS problem (9), or more precisely (using the previously defined simple and double hatted notations),

$\hat{a}(W)=\hat{\hat{a}}(W)$,

$\hat{b}(W)=\hat{\hat{b}}(W)$.

Condition (14) means that the weighting matrix $W$ equalizes the eigenvalues of $\Phi^{T} W \Phi$. For such special weighting matrices, the TSA is thus perfectly justified. However, the situation has been unclear for other weighting matrices, including the identity matrix (corresponding to the unweighted case).

\subsection{The unweighted TSA}

Theorem 1 answers the optimality question about the TSA only in the case of a special class of weighting matrices $W$ satisfying (14). However, in some other works (e.g., Gomez and Baeyens (2004, 2005), McKelvey and Hanner (2003), Mzyk (2000), Palanthandalam-Madapusi et al. $(2006,2005))$, the TSA is typically applied with the unweighted LS solution in its first stage, corresponding to the use of the identity matrix for the weighting matrix. It is clear that usually the identity weighting matrix does not satisfy condition (14). There thus appears to be a contradiction between the established optimality of the algorithm and the usual practice. The purpose of this subsection is to resolve this "contradiction" by justifying the use of the unweighted LS solution in the TSA.

For the ease of presentation, the TSA using the unweighted LS solution in its first stage will be referred to as the Unweighted TSA 
in the sequel. Hence, the first stage of the algorithm in this case consists in computing the unweighted LS solution $\left(I_{N}\right.$ is the $N \times N$ identity matrix)

$\hat{\theta}\left(I_{N}\right)=\left(\Phi^{T} \Phi\right)^{-1} \Phi^{T} Y$,

while the second stage is the same as (12) and (13).

Though each stage of the Unweighted TSA is optimal for its own purpose, it is not obvious if the overall algorithm results in a solution which is optimal in any sense. The following result provides an answer to this question.

Theorem 2. The Unweighted TSA produces the optimal solution of the nonlinear $L S$ problem (9), if the weighting matrix $W$ of this latter is chosen to be

$\check{W}=\alpha\left(\Phi \Phi^{T}\right)^{+}$

where $\alpha$ is any positive scalar and $\left(\Phi \Phi^{T}\right)^{+}$is the Moore-Penrose pseudo-inverse of the matrix $\left(\Phi \Phi^{T}\right)$. In other words, for such a special W,

$\hat{\hat{a}}\left(I_{N}\right)=\hat{a}(\check{W})$

$\hat{\hat{b}}\left(I_{N}\right)=\hat{b}(\check{W})$

where the hatted notations have been defined in Notes on Notations in Section 3.1.

The proof of this theorem will need the following classical result which can be found in textbooks on matrices, for instance in Barnet (1990).

Lemma 1. For any two matrices $A \in \mathbb{R}^{m \times l}$ and $B \in \mathbb{R}^{l \times n}$, both of rank $l$, the equality

$(A B)^{+}=B^{T}\left(B B^{T}\right)^{-1}\left(A^{T} A\right)^{-1} A^{T}$

holds, where $(A B)^{+}$is the Moore-Penrose pseudo-inverse of the matrix $(A B)$.

Proof of Theorem 2. Assumption 2 implies that $\Phi$ has full column rank. The application of Lemma 1 with $A=\Phi$ and $B=\Phi^{T}$ then leads to

$$
\begin{aligned}
\Phi^{T} \breve{W} \Phi & =\Phi^{T}\left(\alpha\left(\Phi \Phi^{T}\right)^{+}\right) \Phi \\
& =\Phi^{T}\left(\alpha \Phi\left(\Phi^{T} \Phi\right)^{-1}\left(\Phi^{T} \Phi\right)^{-1} \Phi^{T}\right) \Phi \\
& =\alpha I_{l} .
\end{aligned}
$$

According to Theorem 1, when such a weighting matrix $\breve{W}$ is used in the TSA, it produces the optimal solution of the nonlinear LS problem (9) defined with the same weighting matrix $\breve{W}$, or more formally,

$$
\begin{aligned}
& \hat{a}(\check{W})=\hat{\hat{a}}(\check{W}) \\
& \hat{b}(\check{W})=\hat{\hat{b}}(\check{W}) .
\end{aligned}
$$

Now let us examine the first stage of the TSA. When it uses the weighting matrix $\check{W}=\alpha\left(\Phi \Phi^{T}\right)^{+}$, the unconstrained LS solution is, following (11) and then (19),

$$
\begin{aligned}
\hat{\theta}(\check{W}) & =\left(\Phi^{T} \check{W} \Phi\right)^{-1} \Phi^{T} \check{W} Y \\
& =\left(\alpha I_{l}\right)^{-1} \Phi^{T}\left(\alpha\left(\Phi \Phi^{T}\right)^{+}\right) Y .
\end{aligned}
$$

Again apply Lemma 1 with $A=\Phi$ and $B=\Phi^{T}$, then

$$
\begin{aligned}
\hat{\theta}(\check{W}) & =\left(\alpha I_{l}\right)^{-1} \Phi^{T}\left(\alpha \Phi\left(\Phi^{T} \Phi\right)^{-1}\left(\Phi^{T} \Phi\right)^{-1} \Phi^{T}\right) Y \\
& =\left(\Phi^{T} \Phi\right)^{-1} \Phi^{T} Y .
\end{aligned}
$$

The last result is nothing but the unweighted LS solution $\hat{\theta}\left(I_{N}\right)$ as defined in equation (15). It then follows that $\hat{\theta}(\check{W})=\hat{\theta}\left(I_{N}\right)$. This result indicates that the first stage of the TSA produces the same result with the weighting matrix $\breve{W}$ or $I_{N}$. As the second stage does not involve the weighting matrix, the TSA produces the same result with these two weighting matrices, or more formally,

$$
\begin{aligned}
& \hat{\hat{a}}\left(I_{N}\right)=\hat{\hat{a}}(\check{W}) \\
& \hat{\hat{b}}\left(I_{N}\right)=\hat{\hat{b}}(\check{W}) .
\end{aligned}
$$

This result and (20) then lead to

$$
\begin{aligned}
& \hat{\hat{a}}\left(I_{N}\right)=\hat{a}(\check{W}) \\
& \hat{\hat{b}}\left(I_{N}\right)=\hat{b}(\check{W}),
\end{aligned}
$$

which complete the proof.

The above result shows that using the unweighted LS solution in the TSA does solve the nonlinear LS problem (9), provided this nonlinear LS problem is formulated with a particular weighting matrix $\breve{W}$ which is different from the identity matrix. This provides a theoretical justification of the Unweighted TSA. As a matter of fact, the use of the unweighted LS solution is well in line with the assumed additive white noise of the considered system; the use of any non-diagonal weighting matrix would color the noise, which is usually not a desired effect.

\subsection{Dealing with colored noises}

If the additive noise $v(t)$ in (2) is colored instead of being white, the Unweighted TSA still yields consistent estimates, because the regressor $\tilde{\Phi}(t)$ in (5) is uncorrelated with $v(t)$ for an FIR transfer function $G(q)$. However, the estimates are less efficient. In other words, there is a choice of the weighting matrix $W$ for the TSA leading to lower estimation uncertainties.

In order to better deal with colored noises, it is important to distinguish two weighting matrices, one is used in the first stage of the TSA, the other in the weighted nonlinear LS problem (9). As a matter of fact, the TSA using an arbitrary weighting matrix in its first stage (11) solves the weighted nonlinear LS problem (9) which is formulated with another weighting matrix, as stated in the following theorem.

Theorem 3. The TSA using any symmetric positive definite weighting matrix $W \in \mathbb{R}^{N \times N}$ in its first stage (11) produces the optimal solution of the nonlinear LS problem (9), if in this latter the weighting matrix $W$ is replaced by

$\tilde{W}=\alpha W^{\frac{1}{2}}\left(W^{\frac{1}{2}} \Phi \Phi^{T} W^{\frac{1}{2}}\right)^{+} W^{\frac{1}{2}}$.

Here $\alpha$ is any positive scalar, $W^{\frac{1}{2}}$ is the symmetric matrix square root of $W$, and $M^{+}$denotes the Moore-Penrose pseudo-inverse of the matrix M. More formally, for such a special $\tilde{W}$,

$$
\begin{aligned}
& \hat{\hat{a}}(W)=\hat{a}(\tilde{W}) \\
& \hat{\hat{b}}(W)=\hat{b}(\tilde{W}) .
\end{aligned}
$$

See again Notes on Notations in Section 3.1 for the hatted notations.

Proof of Theorem 3. The proof of this result is very similar to that of Theorem 2, the only notable difference is that now Lemma 1 is applied with $A=W^{\frac{1}{2}} \Phi$ and $B=\Phi^{T} W^{\frac{1}{2}}$. Hence here the proof is only sketched. It is first shown that $\Phi^{T} \tilde{W} \Phi=\alpha I_{l}$ and consequently, according to Theorem 1 ,

$$
\begin{aligned}
& \hat{a}(\tilde{W})=\hat{\hat{a}}(\tilde{W}) \\
& \hat{b}(\tilde{W})=\hat{\hat{b}}(\tilde{W}) .
\end{aligned}
$$


It is then shown that for the first stage of the TSA,

$\hat{\theta}(\tilde{W})=\hat{\theta}(W)$

and it follows that

$\hat{\hat{a}}(W)=\hat{\hat{a}}(\tilde{W})$

$\hat{\hat{b}}(W)=\hat{\hat{b}}(\tilde{W})$.

Finally, the two sets of equalities (22) and (23) imply

$\hat{\hat{a}}(W)=\hat{a}(\tilde{W})$

$\hat{\hat{b}}(W)=\hat{b}(\tilde{W})$.

For colored noises, the optimal choice of $W$ in the TSA is still unknown to our knowledge. However, the first stage of the TSA ignores the rank constraint (10b) and considers the weighted LS problem (10a) only, which suggests that $W$ could be chosen as the inverse of the noise covariance matrix, according to the principle of the best linear unbiased estimate (see e.g., Söderström and Stoica (1989)). That is, we choose $W$ to be the inverse of the covariance matrix $R$ of $v$ in (4) which writes, for the finite data set $\{u(t), y(t)\}_{t=1}^{N}$,

$R:=E\left\{V V^{T}\right\}$,

where $V$ is defined in (6) and $E$ means the mathematical expectation.

If the TSA takes $W=R^{-1}$, Theorem 3 says that the resulting estimates are the solution of the weighted nonlinear LS problem (9) where $W$ is replaced by

$\tilde{W}=R^{-\frac{1}{2}}\left(R^{-\frac{1}{2}} \Phi \Phi^{T} R^{-\frac{1}{2}}\right)^{+} R^{-\frac{1}{2}}$.

In practice, the noise $v(t)$ and the noise covariance matrix $R$ are normally unknown and have to be estimated. An efficient method for estimating such an inverse noise covariance matrix was recently given by David and Bastin (2001), where $v(t)$ is treated as an auto-regressive (AR) process, and the inversion of the large noise covariance matrix $R$ is avoided by using the AR model of the noise. When this approach is adopted in the first stage of the TSA, an unweighted LS estimation $\hat{\theta}\left(I_{N}\right)$ is first estimated, which is used to estimate the noise $v(t)$. Next, the estimated noise sequence is modeled as an AR process in order to compute the first estimate of the noise covariance inverse $R_{1}^{-1}$, which is used to compute the weighted LS solution $\hat{\theta}\left(R_{1}^{-1}\right)$. A new noise sequence is then estimated with the new model, resulting in a new estimate of the noise covariance inverse $R_{2}^{-1}$, and so on. See David and Bastin (2001) for more details.

The iteration between the estimation of $R^{-1}$ and that of $\hat{\theta}\left(R^{-1}\right)$ is not guaranteed to be convergent, as pointed out in David and Bastin (2001); however, significant improvements are often observed after a few iterations. The effectiveness of the TSA using this method for noise covariance estimation will be demonstrated in Example 2.

\section{Examples}

This section presents two numerical examples to verify the theoretical analysis in Section 3.

Example 1. For the Hammerstein model in Fig. 1,

$$
\begin{aligned}
y(t) & =G(q) x(t)+v(t) \\
& =\left(0.4472 q^{-1}-0.8944 q^{-2}\right) x(t)+v(t), \\
x(t) & =u(t)+2 u^{2}(t)+5 u^{3}(t)+7 u^{4}(t)+u^{5}(t) .
\end{aligned}
$$

Table 1

Estimates of $b=[0.4472,-0.8944]^{T}$ in the second row, estimates of $a=$ $[1,2,5,7,1]^{T}$ in the third row, and associated loss function $L(b, a, W)$ in the bottom row.

\begin{tabular}{lll}
\hline $\begin{array}{l}W=I \\
\text { TSA }\end{array}$ & $\begin{array}{l}W=\breve{W} \text { as in (16) } \\
\text { TSA }\end{array}$ & $\begin{array}{l}W=\check{W} \text { as in }(16) \\
\text { lsqnonlin }\end{array}$ \\
\hline$\left[\begin{array}{c}0.4473 \pm 0.0013 \\
-0.8944 \pm 0.0006\end{array}\right]$ & {$\left[\begin{array}{c}0.4473 \pm 0.0013 \\
-0.8944 \pm 0.0006\end{array}\right]$} & {$\left[\begin{array}{c}0.4473 \pm 0.0015 \\
-0.8944 \pm 0.0019\end{array}\right]$} \\
{$\left[\begin{array}{l}0.9895 \pm 0.1599 \\
1.9932 \pm 0.0777 \\
5.0016 \pm 0.0477 \\
7.0004 \pm 0.0058 \\
0.9998 \pm 0.0027\end{array}\right]$} & {$\left[\begin{array}{l}0.9895 \pm 0.1599 \\
1.9932 \pm 0.0777 \\
5.0016 \pm 0.0477 \\
7.0004 \pm 0.0058 \\
0.9998 \pm 0.0027\end{array}\right]$} & {$\left[\begin{array}{l}0.9897 \pm 0.1616 \\
1.9931 \pm 0.0781 \\
5.0014 \pm 0.0383 \\
7.0002 \pm 0.0128 \\
0.9997 \pm 0.0045\end{array}\right]$} \\
\hline 223.2076 & 0.0302 & 0.0302 \\
\hline
\end{tabular}

The noise $v(t)$ is a zero-mean white Gaussian noise with variance $\sigma_{v}^{2}$. Here $b=\left[\begin{array}{ll}0.4472 & -0.8944\end{array}\right]^{T}$ satisfies Assumption 1 so that the parameterization of $a$ and $b$ is unique. The input $u(t)$ is generated by passing a fixed realization of uniformly-distributed processes with magnitude range $[-3,3]$ through the filter $1 /(1-$ $\left.0.5 q^{-1}\right)$, so that $u(t)$ covers sufficient nonlinear ranges of the input nonlinearity. 100 Monte Carlo simulations are implemented, where each simulation takes a different realization ${ }^{2}$ of $v(t)$.

Two groups of estimates of $a$ and $b$ are obtained by the TSA using $\breve{W}$ in (16) and by the Unweighted TSA. Another group of estimates is obtained by directly solving the nonlinear weighted LS problem (9) with $\breve{W}$ in (16) using the "Isqnonlin" function of the Matlab Optimization Toolbox. The nonlinear LS problem is sensitive to initial estimates; hence the true parameters $a$ and $b$ are used to initiate "lsqnonlin" for the purpose of comparison. Table 1 list the mean and the standard deviations of the estimates in 100 Monte Carlo simulations, for the noise level $\sigma_{v}^{2}=1$. The means of the associated loss functions $L(b, a, W)$ defined in (9) are listed as well at the bottom row in Table 1. In each simulation, 100 data points of $u(t)$ and $y(t)$ are collected, i.e., $N=100$.

In Table 1, the two groups of estimates from the TSA using $W=I_{N}$ and from that using $W=\breve{W}$ as in (16) are exactly the same, which is consistent with Theorem 2 . The estimates appear consistent, as proved Theorem 2.1 in Bai (1998). Since "Isqnonlin" takes the luxury of using the true parameters $a$ and $b$ as the initial estimates, the global optima are expected. The resulting estimates are close to the counterparts from the TSA using $W=\breve{W}$ as in (16); in fact, the corresponding loss functions $L(b, a, W)$ in the last two columns are exactly the same, which validates the conclusion in Theorem 1. On the other hand, if "Isqnonlin" is applied for $W=I$, the loss function $L(b, a, W)$ is around 94.6930, smaller than the counterpart of TSA (223.2076, the first element in the last row of Table 1). This says that the Unweighted TSA does not find the solution of the unweighted nonlinear LS optimization problem, i.e.,

$\min _{b, a} \frac{1}{2}\left(\Phi \overline{b a^{T}}-Y\right)^{T}\left(\Phi \overline{b a^{T}}-Y\right)$.

Instead, it provides the solution of the weighted nonlinear LS optimization problem (9) with $W=\breve{W}$ as in (16), as indicated by the fact that the two groups of estimates from the TSA using $W=I_{N}$ and from that using $W=\breve{W}$ as in (16) are exactly the same. This is consistent with Theorem 2.

\footnotetext{
2 The uniform random generator state in Matlab for $u(t)$ is fixed to 1 , while the Gaussian random generator states for $v(t)$ are fixed to $[1,2, \ldots, 100]$, so that the simulation results can be reproduced.
} 
Table 2

Estimates of $a$ and $b$ for colored noises.

\begin{tabular}{cll}
\hline$\sigma_{e}^{2}=1$ & $W=I$ & $W=\hat{R}^{-1}$ \\
\hline$b=\left[\begin{array}{c}0.4472 \\
-0.8944\end{array}\right]$ & {$\left[\begin{array}{c}0.4477 \pm 0.0043 \\
-0.8942 \pm 0.0022\end{array}\right]$} & {$\left[\begin{array}{c}0.4474 \pm 0.0017 \\
-0.8943 \pm 0.0009\end{array}\right]$} \\
$a=\left[\begin{array}{l}1.0000 \\
2.0000 \\
5.0000 \\
7.0000 \\
1.0000\end{array}\right]$ & {$\left[\begin{array}{l}0.9771 \pm 0.1911 \\
1.9720 \pm 0.1232 \\
4.9980 \pm 0.0601 \\
7.0015 \pm 0.0091 \\
0.9999 \pm 0.0034\end{array}\right]$} & {$\left[\begin{array}{c}0.9948 \pm 0.1085 \\
1.9942 \pm 0.0577 \\
4.9981 \pm 0.0304 \\
7.0002 \pm 0.0040 \\
1.0000 \pm 0.0017\end{array}\right]$} \\
\hline
\end{tabular}

Example 2. All the configurations are the same as Example 1 except that the noise $v(t)$ is generated as

$v(t)=\frac{1}{1-0.9 q^{-1}} e(t)$,

where $e(t)$ is a zero-mean white Gaussian noise with variance $\sigma_{e}^{2}$. That is, $v(t)$ is colored instead of being white.

Table 2 listed the estimates of $a$ and $b$ obtained by the TSA using estimated inverse covariance matrix, as proposed in Section 3.3. In particular, the order of the noise AR model of $v(t)$ is selected according to the FPE criterion based on F statistical test with 95\% confidence level (e.g., see Eq. (4.30) in Söderström and Stoica (1989)); in the 100 Monte Carlo simulations, the order 1 is correctly selected for 94 times, and the order 2 is selected for the rest. Some improvements are observed in the first 3 or 4 iterations between the estimation of $R^{-1}$ and that of $a$ and $b$. As a comparison, another group of estimates of $a$ and $b$ is obtained by the Unweighted TSA. In Table 2, the two groups of estimates appear both consistent, but the estimates of $a$ and $b$ associated with $W=\hat{R}^{-1}$ have much smaller uncertainties, in terms of standard deviations, than those obtained by the Unweighted TSA. This observation is in favor of choosing $W$ equal the inverse of the noise covariance matrix.

\section{Conclusion}

This paper revisited the Two-Stage Algorithm (TSA) proposed by Bai (1998) for identification of Hammerstein systems, and obtained some optimality results of the TSA. The motivation arises from a somewhat contradictory fact: though the optimality of the TSA has been established in Bai (1998) (restated here in Theorem 1) for a special class of weighting matrices satisfying an equality in (14), the TSA, adopted in some other works, usually uses the unweighted LS solution in its first stage. That is, the identity matrix, which usually does not belong to the above special weighting matrices, is used. Theorem 2 of this paper shows that the Unweighted TSA indeed gives the solution of (9) or its equivalence (10) for a particular weighting matrix $\breve{W}$ as given in (16). This provides a theoretical justification of using the Unweighted TSA, and leads to Theorem 3 where a generalization is made to the case of colored noise with noise whitening. Finally, these theoretical results were validated via two simulation examples of identification of Hammerstein systems.

There remain some interesting questions to be answered in future studies. An important one, for instance, is the converse question of Theorem 3. Theorem 3 says that using any symmetric positive definite weighting matrix $W \in \mathbb{R}^{N \times N}$ in the TSA produces the optimal solution of the nonlinear LS problem (9) with its weighting matrix replaced by $\tilde{W}$ as defined in (21). The converse question is: for any symmetric weighting matrix $W_{N L S}$ used in the nonlinear LS problem (9), does there always exist a weighting matrix $W_{\text {TSA }}$ for the TSA to solve the given nonlinear LS problem? While the answer to this question appears to be negative, it is not obvious how to characterize the set of all weighting matrices $W_{N L S}$ such that the related nonlinear LS problem can be solved by the TSA with an appropriate weighting matrix $W_{\text {TSA }}$.

\section{References}

Abrahamsson, R., Kay, S., \& Stoica, P. (2007). Estimation of the parameters of a bilinear model with applications to submarine detection and system identification. Digital Signal Processing, 17, 756-773.

Bai, E. (1998). An optimal two-stage identification algorithm for Hammerstein-Wiener nonlinear systems. Automatica, 34(3), 333-338.

Bai, E., \& Liu, Y. (2006). Least squares solutions of bilinear equations. Systems $\mathcal{E}$ Control Letters, 55, 466-472.

Balestrino, A., Landi, A., Ould-Zmirli, M., \& Sani, L. (2001). Automatic nonlinear autotuning method for Hammerstein modeling of electrical drives. IEEE Transactions on Industrial Electronics, 48(3), 645-655.

Barnet, S. (1990). Oxford applied mathematics and computing sciences series, Matrices methods and applications. Oxford: Clarendon Press.

Chang, F., \& Luus, R. (1971). A noniterative method for identification using Hammerstein model. IEEE Transactions on Automatic Control, 16(5), 464-468.

Cohen, S., \& Tomasi, C. (1997). Systems of bilinear equations. Computer Science Dept., Stanford Univ., Stanford, CA.

David, B., \& Bastin, G. (2001). An estimator of the inverse covariance matrix and its application to $\mathrm{ml}$ parameter estimation in dynamical systems. Automatica, 37(1), 99-106.

Dempsey, E., \& Westwick, D. (2004). Identification of Hammerstein models with cubic spline nonlinearities. IEEE Transactions on Biomedical Engineering, 51(2), 237-245.

Eskinat, E., Johnson, S., \& Luyben, W. (1991). Use of Hammerstein models in identification of nonlinear systems. AIChE Journal, 37(2), 255-268.

Goethals, I., Pelckmans, K., Suykens, J., \& Moor, B. D. (2005). Identification of mimo Hammerstein models using least squares support vector machine. Automatica, $41,1263-1272$.

Gomez, J., \& Baeyens, E. (2004). Identification of block-oriented nonlinear systems using orthonormal bases. Journal of Process Control, 14(6), 685-697.

Gomez, J. \& Baeyens, E. (2005). Subspace-based identification algorithms for Hammerstein and wiener models. European Journal of Control, 11, 127-136.

Haber, R., \& Keviczky, L. (1999). Nonlinear system identification: Input-output modeling approach. Dordrecht: Kluwer Academic Publishers.

Janczak, A. (2005). Identification of nonlinear systems using neural networks and polynomial models: A block-oriented approach. New York: Springer-Verlag.

Jurado, F. (2006). A method for the identification of solid oxide fuel cells using a Hammerstein model. Journal of Power Sources, 154(1), 145-152.

Ljung, L. (1999). System identification: Theory for the user (2nd ed.). Englewood Cliffs, NJ: Prentice Hall.

McKelvey, T., \& Hanner, C. (2003). On identification of Hammerstein systems using excitation with a finite number of levels. In Proc. of the 13th international symp. on system identification (SYSID2003) (pp. 57-60).

Mzyk, G. (2000). Application of instrumental variable method to the identification of Hammerstein-Wiener systems. In 6th international conf. MMAR, Miedzyzdroje.

Palanthandalam-Madapusi, H., Bernstein, D., \& Ridley, A. (2006). Subspace identification of periodically switching Hammerstein-Wiener models for magnetospheric dynamics. In 14th IFAC symp. on system identification Newcastle, Australia, (pp. 535-540).

Palanthandalam-Madapusi, H., Ridley, A., \& Bernstein, D. (2005). Identification and prediction of ionospheric dynamics using a Hammerstein-Wiener model with radial basis functions. In 2005 American control conf. Portland, OR, USA (pp. 5052-5057).

Söderström, T., \& Stoica, P. (1989). System identification. London: Prentice Hall.

Srinivasan, R., Rengaswamy, R., Narasimhan, S., \& Miller, R. (2005). Control loop performance assessment. 2. Hammerstein model approach for stiction diagnosis. Industrial \&' Engineering Chemistry Research, 44(17), 6719-6728.

Sung, S. (2002). System identification method for Hammerstein processes. Industrial E Engineering Chemistry Research, 41(17), 4295-4302.

Wang, J., Sano, A., Chen, T., \& Huang, B. (2009). Identification of Hammerstein systems without explicit parameterization of nonlinearity. International Journal of Control, 82(5), 937-952.

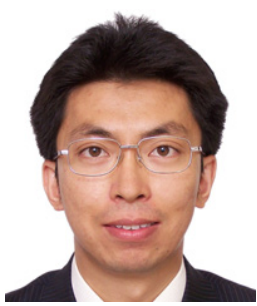

Jiandong Wang received the B.E. degree in automatic control from Beijing University of Chemical Technology, Beijing, China, in 1997, and the M.Sc and Ph.D. degrees from the University of Alberta, Edmonton, AB, Canada, in 2003 and 2007, respectively, all in Electrical and Computer Engineering. From 1997 to 2001, he was a Control Engineer with the Beijing Tsinghua Energy Simulation Company, Beijing, China. From Feb. 2006 to Aug. 2006, he was a Visiting Scholar at the Department of System Design Engineering at the Keio University, Japan. Since December 2006, he has been an Assistant Professor with the Department of Industrial Engineering and Management in the College of Engineering at the Peking University, China. His current research interests include system identification, networked systems, signal processing, process monitoring and management, and their applications to industrial problems. Dr. Wang received 
Peking University P\&G Fellowship for 2008 and a Fellowship from the Japan Society for the Promotion of Science for 2006. He was a recipient of the Izzak Walton Killam Memorial Scholarship, the Alberta Ingenuity Ph.D. Studentship, and the iCore Graduate Student Scholarship at the University of Alberta.

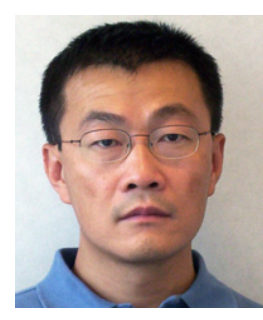

processing.
Qinghua Zhang received his B.S. in 1986 from the University of Science and Technology of China, D.E.A. in 1988 from Université de Lille 1, France, Ph.D. in 1991 and H.D.R. in 1999 both from Université de Rennes 1, France. During 1992 he was a post-doc fellow in Linköping University, Sweden. Since 1993 he works at Institut National de Recherche en Informatique et en Automatique (INRIA) and also at Institut de Recherche en Informatique et Systmes Aléatoires (IRISA), located in Rennes, France, as a research scientist. His main research interests are nonlinear system identification, fault diagnosis and biomedical signal

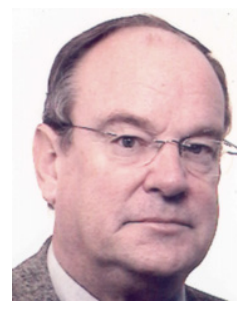

Lennart Ljung received his Ph.D. in Automatic Control from Lund Institute of Technology in 1974. Since 1976 he is Professor of the chair of Automatic Control In Linköping, Sweden, and is currently Director of the Strategic Research Center "Modeling, Visualization and Information Integration" (MOVIII). He has held visiting positions at Stanford and MIT and has written several books on System Identification and Estimation. He is an IEEE Fellow, an IFAC Fellow and an IFAC Advisor as well as a member of the Royal Swedish Academy of Sciences (KVA), a member of the Royal Swedish Academy of Engineering Sciences (IVA), an Honorary Member of the Hungarian Academy of Engineering and a Foreign Associate of the US National Academy of Engineering (NAE). He has received honorary doctorates from the Baltic State Technical University in St Petersburg, from Uppsala University, Sweden, from the Technical University of Troyes, France, from the Catholic University of Leuven, Belgium and from Helsinki University of Technology, Finland. In 2002 he received the Quazza Medal from IFAC, in 2003 he received the Hendrik W. Bode Lecture Prize from the IEEE Control Systems Society, and he was the recipient of the IEEE Control Systems Award for 2007. 


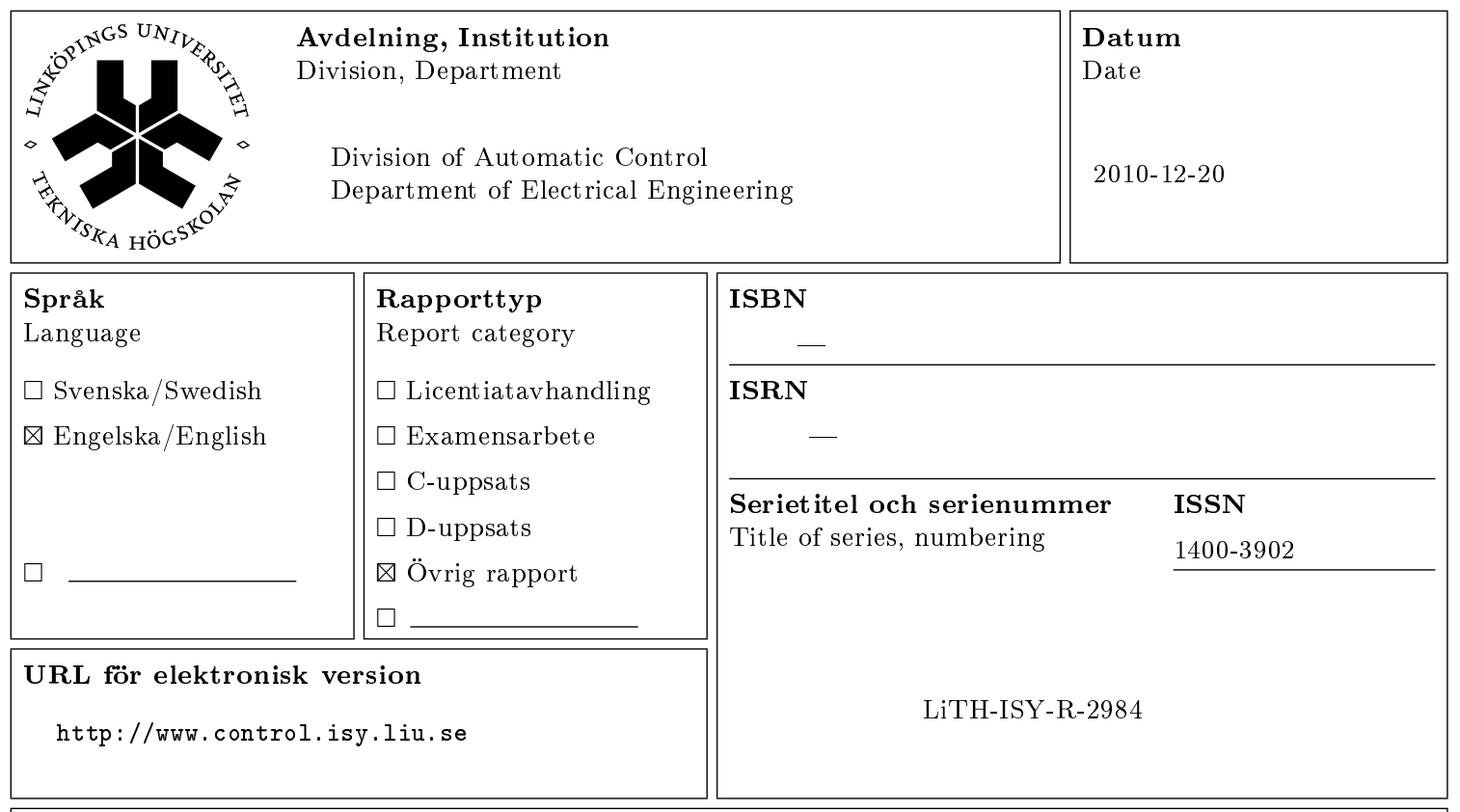

Titel Revisiting Hammerstein system identification through the Two-Stage Algorithm for bilinear Title parameter estimation

Författare Jiandong Wang, Qinghua Zhang, Lennart Ljung

Author

\section{Sammanfattning}

Abstract

The Two-Stage Algorithm (TSA) has been extensively used and adapted for the identification of Hammerstein systems. It is essentially based on a particular formulation of Hammerstein systems in the form of bilinearly parameterized linear regressions. This paper has been motivated by a somewhat contradictory fact: though the optimality of the TSA has been established by Bai in 1998 only in the case of some special weighting matrices, the unweighted TSA is usually used in practice. It is shown in this paper that the unweighted TSA indeed gives the optimal solution of the weighted nonlinear least squares problem formulated with a particular weighting matrix. This provides a theoretical justification of the unweighted TSA, and also leads to a generalization of the obtained result to the case of colored noise with noise whitening. Numerical examples of identification of Hammerstein systems are presented to validate the theoretical analysis. 